\title{
Effect of Inclined Rowing Machine on FES-Assisted Indoor Rowing Exercise Performance
}

\author{
Z. Hussain, M. O. Tokhi, R. Jailani, S. Ahmad and B. S. Ksm. Kader Ibrahim \\ Department of Automatic Control and System Engineering \\ The University of Sheffield, UK \\ cop06zh@sheffield.ac.uk
}

\begin{abstract}
This paper describes the effect of inclined track in an indoor rowing machine on the rowing exercise for paraplegics. The indoor rowing exercise is introduced as a total body exercise for rehabilitation of function of lower extremities through the application of functional electrical stimulation (FES). A model of the machine is developed using the Visual Nastran (Vn4D) software environment. Nine different degrees of inclination are set. Fuzzy logic control is implemented to control the knee and elbow trajectories for each of the inclination angle. The generated level of electrical stimulations for activation of quadriceps and hamstrings muscles are recorded and analysed. The results show that the highest efficiency is achieved at $7^{\circ}$ of inclination. In view of good results obtained, it is concluded that different angles of track inclination significantly affect the level of electrical stimulation required to assist paraplegics' indoor rowing exercise.
\end{abstract}

\section{Keywords - FES; Rowing Exercise; Fuzzy logic control}

\section{INTRODUCTION}

FES-assisted indoor rowing exercise is a hybrid FES activity for rehabilitation of function of lower body for a person with spinal cord injury (SCI). It is introduced as a high intensity, safe, affordable and natural alternative total body exercise [1-4]. It is an enhanced physical activity through the application of functional electrical stimulation (FES) to assist lower body movement that is combined with the voluntary upper body movement. The development of FES-assisted indoor rowing exercise has become increasingly important as an alternative FES hybrid exercise [5]-[9]. In FES-assisted indoor rowing exercise, FES is applied to the muscles for extension and flexion of hip, knee and ankle to perform the rowing exercise. Suitable electrical stimulation to the muscle are required in achieving a smooth and well coordinated rowing manoeuvre.

Similar to other hybrid FES activity, the performance of FES-assisted rowing exercise can be enhanced through the application of assisting devices, exercise technique and the implementation of efficient control strategy. Gharooni et al. [10] introduced an energy storage device, an elastic cable, to reduce the electrical stimulation to hamstrings muscle during the recovery phase. By using the assisting devices, the overall power can be greatly increased, loss of power due to muscle fatigue can be compensated for and the movement maintained even at very low leg power levels.
The performance of FES-assisted indoor rowing exercise can be enhanced even without any additional devices. The rowing machine arrangement and rowing technique can play important roles in achieving better rowing performance. Wang et al. [11] have investigated the effect of inclined ergometer on able body rowing performance. The finding shows that the rowers produced larger range of motion (ROM) in hip and trunk during the rowing cycle but shorter rowing distance and smaller peak force in inclined platform. This paper therefore presents the effect of increasing the degree of inclination of track of indoor rowing machine in reducing the level of electrical stimulation required in assisting paraplegics' rowing exercise. Nine different degrees of inclination (from $1^{\circ}$ to $9^{\circ}$ ) were tested and evaluated. The models of the indoor rowing machine and humanoid were developed using the Visual Nastran (Vn4D) software environment. A fuzzy logic control (FLC) strategy is also implemented using the Matlab/Simulink environment to automatically control the electrical stimulation required for a smooth rowing manoeuvre with coordination of voluntary upper body movement.

\section{DESCRIPTION OF MODEL}

\section{A. Indoor Rowing Exercise Model}

The indoor rowing exercise model developed comprises two parts: rowing machine and humanoid model. They are designed using the Vn4D environment. The humanoid model was developed using the anthropometric data based on Winter's work, and the details are provided in previous work [12]. The machine is designed based on the modified indoor rowing machine that incorporates all basic parts of the machine. A flywheel attached with sprocket is designed to provide damper resistance for pulling phase. A new seating system with high backrest to stabilize the trunk was developed. The seat is attached to a rail (on supporting frame) via sliding constraint with two adjustable safety stops, installed on the rail to limit the motion for knee joint protection against hyperextension and hyperflexion so as to achieve smooth horizontal motion. One end of the supporting frame (includes rail) is attached to front stand base frame by revolute constraint as a hinge to allow the supporting frame to rotate. The other end of the supporting frame is attached to a linear actuator as lifting element. Moreover, the actuator is pivotally interposed between the 
back stand base frame and the supporting frame. The actuator will lift up the supporting frame to different heights of $0.02 \mathrm{~m}$ to $0.18 \mathrm{~m}$ to provide different angle of inclinations $\left(1^{\circ}\right.$ to $\left.9^{\circ}\right)$. Fig. 1 shows the schematic diagram of the system with inclined frame. Fig.2 shows the indoor rowing exercise developed using Vn4D.

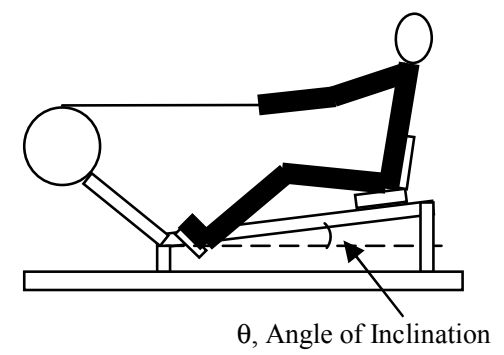

Figure1. Schematic diagram of indoor rowing system with inclined frame

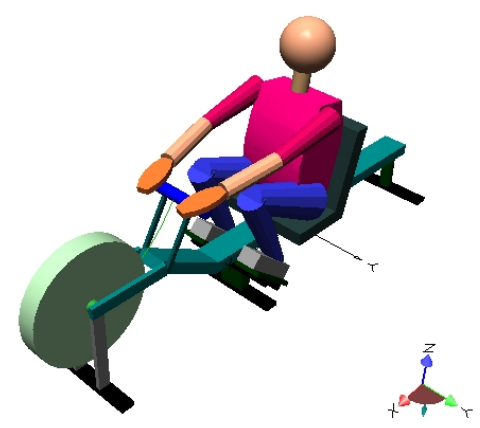

Figure 2. Indoor rowing machine with humanoid model

\section{B. Physiological Based Muscle Model}

In order to simulate FES, the physiological based muscle model for indoor rowing exercise was constructed based on the work of Riener and Fuhr [13]. The model describes the major properties of muscle and segmental dynamics of human during FES-assisted indoor rowing exercise. Two groups of muscle for knee extension (quadriceps) and flexion (hamstrings) are developed. The muscle model developed is composed of three parts, namely muscle activation, muscle contraction and body segmental dynamics. The muscle activation model comprises four main components: recruitment characteristic, frequency characteristic, calcium dynamics and muscle fatigue. The muscle contraction accounts for the force-length property, $f_{f l}$ and force-velocity property, $f_{f v}$, of the muscle and scales the muscle activation, act, by maximum isometric muscle force, $F_{\text {Max }}$, in order to obtain the absolute muscle force. The active joint moment for each muscle is then obtained from the product of moment arm and muscle force. The passive muscle properties are divided into two components: passive elastics, $M_{e l a, j}$ and passive viscous joint moments, $M_{v i s, j}$. The specific parameters of the muscle and independent parameters of muscle and the performance of the developed muscle model in indoor rowing exercise are described in [14].

\section{Implementation of Control Strategies}

A specific control strategy is required to regulate the stimulated pulse width required by muscle model in order to obtain smooth rowing manoeuvre. In this study, four fuzzy logic controllers are designed to control knee extensors and flexors for both legs. They are divided into four phases: main extension phase where quadriceps muscle is activated to drive the rowing backward, resistance extension phase where hamstrings muscle is activated to slow down the rowing backward manoeuvre, main flexion where hamstrings muscle is activated for recovery phase and resistance flexion phase to slow down the forward rowing manoeuvre by activating the quadriceps muscle. There are four inputs selected for the controller. These are the error (difference between actual knee trajectory and reference knee trajectory), change of error and knee angular velocity and acceleration. The error and change of error are measured from the Vn4D indoor rowing exercise simulation model as the system outputs. The output is the stimulation pulse width. Five Gaussian type membership functions are used for each input, error and change of error and five membership functions for the output. Table 1 shows the fuzzy rules for the main extension.

TABLE 1 FUZZY RULES

\begin{tabular}{|c|c|c|c|c|c|}
\hline de & NB & NS & Z & PS & PB \\
\hline NB & VH & VH & HG & MD & LW \\
\hline NS & VH & HG & MD & MD & LW \\
\hline Z & HG & MD & MD & LW & VL \\
\hline PS & MD & MD & LW & VL & VL \\
\hline PB & MD & LW & VL & VL & VL \\
\hline
\end{tabular}

\section{RESULTS AND DISCUSSION}

The effect of the inclined rowing machine in assisting the paraplegics' rowing exercise was evaluated. Different heights of supporting frame were set up to provide different angles of inclination. By adjusting the length of linear actuator, the angles of inclination in the range of $1^{\circ}$ to $9^{\circ}$ were obtained.

In this study, the knee and elbow trajectories are controlled (with predefined reference trajectories, Fig. 3) to provide a consistent rowing sequence in all the nine different angles of inclination. The generated level of electrical stimulation (pulse width) and the torque required 
to obtain smooth rowing manoeuvre were recorded and analyzed. It was noted that the FLC approach worked very well for all the nine different angles of inclination.

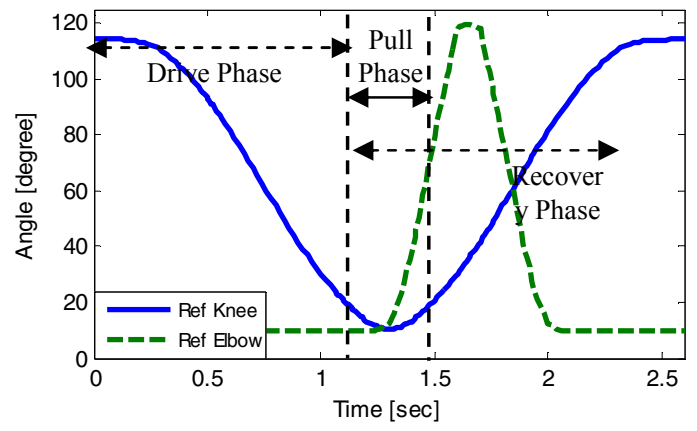

Figure 3. Knee and elbow reference trajectories

The performance of the rowing exercise with different angles of inclination of rowing machine was evaluated from viewpoints of efficiency and amount of electrical stimulation required for rowing manoeuvre. The efficiency of the rowing is calculated from the ratio of the output power and muscle power [15]. Fig.4 shows the efficiency of the rowing exercise at the various angles of inclination. The efficiency increased as the frame inclined higher and the highest efficiency was obtained at $7^{\circ}$.

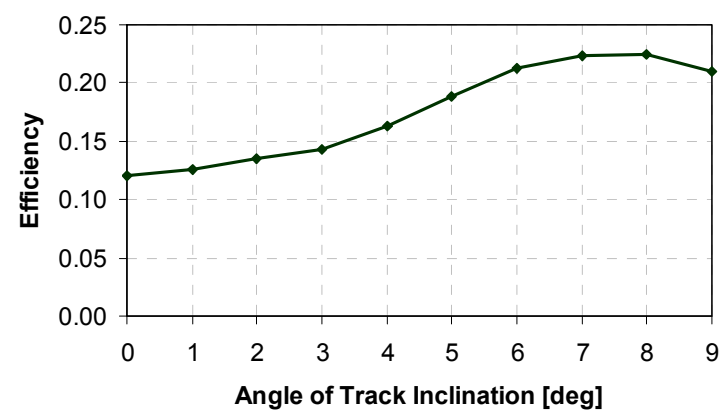

Figure 4. Efficiency vs angle of inclination

The efficiency reduced for angles more than $8^{\circ}$ as higher quadriceps stimulation was required to overcome the gravity. Fig.5 shows the quadriceps and hamstrings stimulation intensity at the various angles of inclination. The stimulation intensity of the hamstrings muscle decreased significantly as the angle of inclination increased. This was due to less hamstring stimulation required during resistance extension phase. The stimulation intensity of quadriceps muscle increased as the angle increased, as higher torque was required to overcome the gravity.

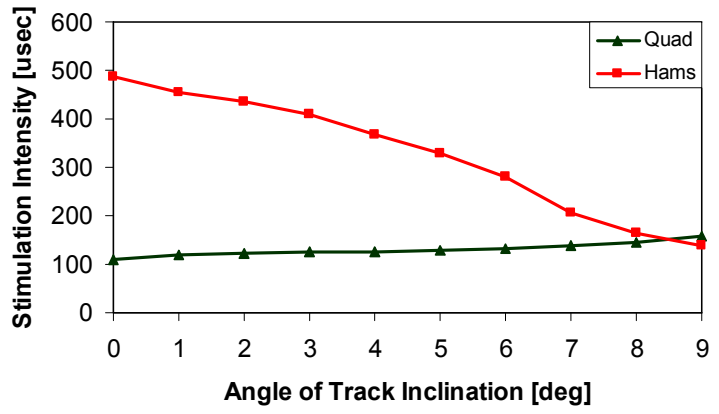

Figure 5. Stimulation intensity vs angle of inclination

Fig.6 shows the average knee torque generated at each of the angles of inclination. It can be seen that the knee torque reduced significantly as the frame inclined higher but increased when the angle was more than $8^{\circ}$.

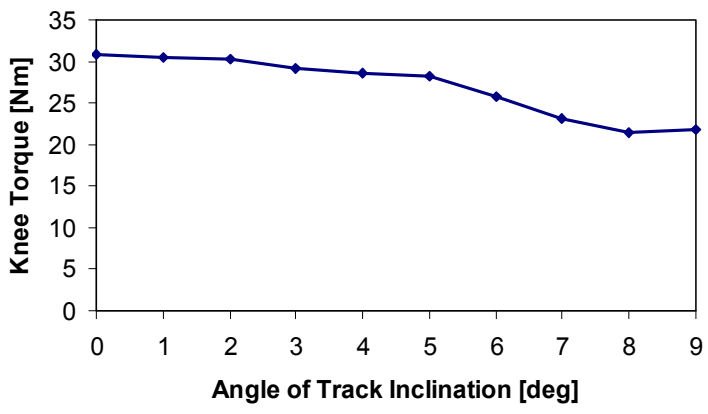

Figure 6. Knee torque vs angle of inclination

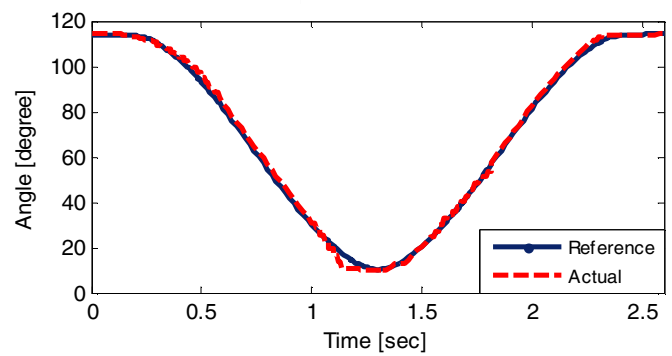

Figure 7. Reference and actual knee trajectories

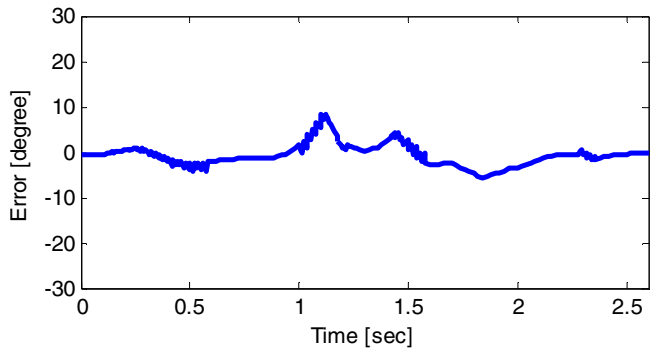

Figure 8. Error in knee trajectory 
Fig.7 shows the actual and reference knee trajectories when the angle of inclination is setup to $7^{\circ}$ and Fig. 8 shows the error after several attempts of FLC tuning. The error between the reference and actual knee trajectory is considered very small for a complex physiological based muscle model with maximum error of 5.3\%. Fig.9 shows the stimulation of quadriceps and hamstrings required performing one stroke rowing manoeuvre at $7^{\circ}$ angle of inclination and Fig.10 is the corresponding knee torque. The average quadriceps and hamstrings muscle stimulation pulse width were $138.3 \mu \mathrm{sec}$ and $206.6 \mu \mathrm{sec}$ respectively. The average knee torque produced was $23.2 \mathrm{Nm}$. Note that the average knee torque obtained was from the average of absolute knee torque taken from the muscle model.

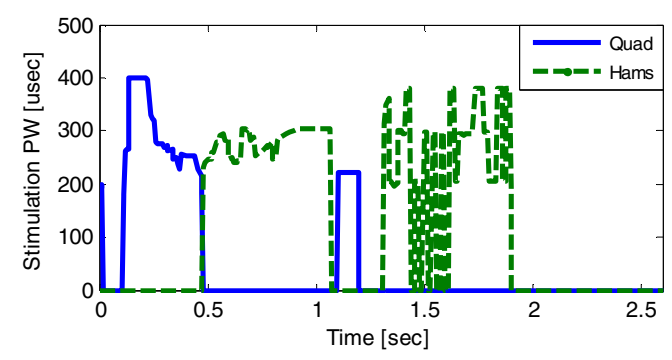

Figure 9. Stimulation pulse width

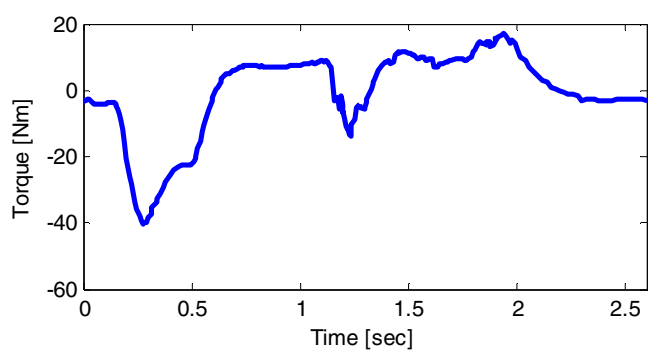

Figure 10. Torque applied to knee joint

\section{CONCLUSION}

The present study has established the performance of FES-assisted indoor rowing exercise with different angles of inclination. The results show that different angles of inclination can significantly impact on assisting indoor rowing exercise for paraplegics by reducing the level of electrical stimulation of hamstrings muscle. It has been noticed that the most efficient rowing exercise can be performed at an angle of inclination of $7^{\circ}$. Furthermore, the torque required to perform the rowing manoeuvre has been suitable, in the range of 0 to $50 \mathrm{Nm}$, and this agreed with previous work. The muscle stimulation can be further reduced by introducing assisting elements such as spring that will be consider in future work.

\section{REFERENCES}

[1] G.D. Wheeler, B. Andrews, R. Lederer, R. Davoodi, K. Natho, C. Weiss, J. Jeon, Y. Bhambhani, and R.D. Steadward, "Functional electric stimulation-assisted rowing: Increasing cardiovascular fitness through functional electric stimulation rowing training in persons with spinal cord injury." Arch Phys Med Rehabil, vol. 83, no. 8, pg. 1093-9, (2002).

[2] R. Davoodi, B.J. Andrews and G.D. Wheeler, "Modified concept $2^{\mathrm{TM}}$ rowing machine with manual FES controller for total body exercise in paraplegia." Proceedings of International FES Society, pg. 114116, 2001.

[3] R. Davoodi, B.J. Andrews and G.D. Wheeler, "Manual and Automatic Control of FES-Assisted Indoor Rowing Exercise." Joint EMBS-BMES Conference, Houston, Texas, USA, pg. 2377-2378, 2002.

[4] R. Davoodi, B.J. Andrews, G.D. Wheeler and R. Lederer, "Development of an indoor rowing machine with manual FES controller for total body exercise in paraplegia." IEEE Trans. Neural Syst. Rehabil. Eng., vol 10, no. 3, pg. 197-203, 2002.

[5] R. Davoodi and B.J. Andrews, "Fuzzy logic control of FES rowing exercise in paraplegia." IEEE Trans Biomed Eng, vol. 51, no. 3, pg. 541-543, 2004.

[6] S.E. Halliday, A.B. Zavatsky and K. Hase, "Can Functional Electric Stimulation-Assisted Rowing Reproduce a Race-Winning Rowing Stroke?” Arch Phys Med Rehabil, vol. 85, pg. 1265-1272, 2004.

[7] B.J. Andrew, K. Hase, S.E. Halliday, A.B. Zavatsk, "Biomechanical Study of FES Rowing and Inclined Bench Sliding Systems Using Simulation Models" 7th Annual Conference of the International FES Society, Slovenia, 2002.

[8] J. Verellen, B. Kerby, B. Olijnyk, S. Saab, G. Smith, J. Jeon, R.D. Steadward, Y. Vanlandewijck, B.J. Andrews, G.D. Wheeler, "A Comparison of the Peak Physical Work Capacity during Arm Ergometry, Fes Cycling and Two Hybrid Exercise Conditions in Spinal Cord Injured" 7th Vienna International Workshop on Functional Electrical Stimulation, Vienna, Austria,2001.

[9] S. Julai and M.O. Tokhi, "Establishing Reference Trajectory of Humanoid Indoor Rowing Exercise" Proceeding Of International Conference On Recent Advances In Mechanical \& Material Engineering, No. 263, Pg 1433-1437, 2005.

[10] S. Gharooni, S. Sareh, and M.O. Tokhi. "Development of FESrowing machine with quadriceps stimulation and energy storing device." $12^{\text {th }}$ Annual Conference of the International FES Society, USA, 2007.

[11] S.Y. Wang, C.C Wu and C.F. Huang, "The effect of inclined ergometer on rowing performance." International Society of Biomechanics XIXth Congress, New Zealand, pg 414, 2003.

[12] Z. Hussain, M.O. Tokhi and S. Gharooni, "Dynamic simulation of indoor rowing exercise for paraplegics", Proceeding of $2^{\text {nd }}$ Asia International Conference on Modelling and Simulation, AMS2008, pg 901-904, 2008.

[13] R. Riener and T. Fuhr. "Patient-driven control of FES-supported standing-up: A simulation study." IEEE Trans.Rehab.Eng.,vol.6, no.2, pp.113-124, 1998.

[14] Z. Hussain and M.O. Tokhi, "Modelling of muscle extension and flexion for FES-assisted indoor rowing exercise", Proceeding of $2^{\text {nd }}$ Asia International Conference on Modelling and Simulation, AMS2008, pg 963-967, 2008.

[15] G.A Gaesser and G.A. Brooks. "Muscular efficiency during steadyrate exercise: effect of speed and work rate." Journal of Applied Physiology, no 38, vol. 6, pg 1132-1136, 1975. 